\title{
Exposure to Phthalate Esters and the Risk of Endometriosis
}

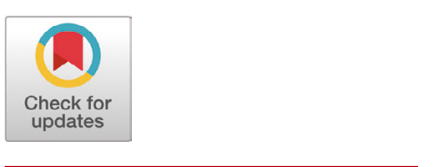

Received: May 7, 2020

Revised: May 20, 2020

Accepted: May 26, 2020

${ }^{\dagger}$ Corresponding author

Sung Hoon Kim

Dept. of Obstetrics and Gynecology

University of Ulsan College of Medicine,

Asan Medical Center, Seoul 05505, Korea.

Tel: +82-2-3010-3647

Fax: $+82-2-2045-4564$

E-mail: kimsung@amc.seoul.kr

Copyright $\odot 2020$ The Korean Society of Developmental Biology.

This is an Open Access article distributed under the terms of the Creative Commons Attribution Non-Commercial License (http://creativecommons.org/licenses/ by-nc/4.0/) which permits unrestricted non-commercial use, distribution, and reproduction in any medium, provided the original work is properly cited.

ORCID

Ju Hee Kim

https://orcid.org/0000-0001-5713-7743

Sung Hoon Kim

https://orcid.org/0000-0003-2567-2092

Conflict of interests

The authors declare no potential conflict of interest.

Acknowledgements

Not applicable.

Authors' contributions

Writing - original draft: JH Kim

Writing - review \& editing: SH Kim

\section{Ethics approval}

This article does not require IRB/IACUC approval because there are no human and animal participants.

\author{
Ju Hee Kim and ${ }^{\dagger}$ Sung Hoon Kim
}

Dept. of Obstetrics and Gynecology, University of Ulsan College of Medicine, Asan Medical Center, Seoul 05505, Korea

\section{Abstract}

Endometriosis is a common gynecologic disease, worldwide, whose true prevalence is uncertain because it is a difficult disease to diagnose. Endometriosis is a common cause of chronic pelvic pain, dysmenorrhea, and infertility, and is also associated with ovarian cancer. Although the risk factors for endometriosis are unclear, there is increasing evidence that exposure to environmental contaminants, especially phthalates, could affect the pathogenesis of endometriosis. Phthalates are industrial chemicals, used to make flexible plastics, and are present in numerous common plastic products, including medical devices and materials. Several in vitro studies have suggested a positive association between exposure to phthalate, or phthalate metabolites, and the risk of endometriosis. Since the 2000s, studies based on human plasma and urinary concentrations of various phthalate metabolites have been published, but there are still limitations to our understanding of the pathophysiology of phthalates and endometriosis. This report aims to review the current state of knowledge about a possible role of phthalates in the pathogenesis of endometriosis based on cell culture, animal models, and human data.

Keywords: Phthalate, DEHP, Endocrine disruptor, Endometriosis

\section{INTRODUCTION}

Endometriosis is defined as the presence of endometrial tissue outside the uterus, mainly in the pelvic cavity (Zondervan et al., 2018). It is an estrogen-dependent disease, related to reproductive age (Kitawaki et al., 2002), whereby endometrial-like cells result in a localized inflammatory response, causing pelvic adhesion (Klemmt \& Starzinski-Powitz, 2018). For complex pathophysiological reasons, endometriosis can induce chronic pelvic pain, dysmenorrhea and infertility, and is also associated with ovarian cancers, such as clear cell or endometrioid carcinomas (Herreros-Villanueva et al., 2019). The worldwide prevalence of endometriosis is approximately $10 \%$ of women of reproductive age (Shafrir et al., 2018), but the true prevalence is uncertain because it is difficult disease to diagnose. Diagnosis of endometriosis follows surgical assessment, but not all patients require surgery because the symptoms are variable, and there are no effective biomarkers available (Zondervan et al., 2020).

Although it is still unclear how endometriosis develops and progress, evidence is growing that exposure to environmental contaminants, especially phthalates, might affect its pathogenesis. Phthalates are diesters of phthalic acid, and are often used in the plastics industry to make plastics flexible and to solubilize other agents. They are present in numerous everyday products, such as plastics, vinyl, car products, ink, soap and shampoo (CDC, 2009). Exposure to phthalates is mainly through ingestion, 
and they are hydrolyzed by the digestive system into a form of monoester, absorbed, oxidized and excreted in urine (Frederiksen et al., 2007). The phthalate diester, di-2-ethylhexyl phthalate (DEHP) is of particular interest and concern because it is found in many plastic products, vinyl materials, furniture, and toys, as well as medical devices, including blood storage bags which have direct exposure to the bloodstream (ATSDR, 2002).

Several animal studies have shown that DEHP can act as an endocrine disruptor and that it affects the reproductive system and fertility, for example, by inducing testicular atrophy, increasing complications in pregnancy and suppressing estrogen production (Lovekamp-Swan \& Davis, 2003; Jarfelt et al., 2005; Schmidt et al., 2012). The fact that DEHP can alter estrogen synthesis has provoked studies on its role in endometriosis, because it is an estrogen-dependent disease. Research based on in vitro studies has suggested a positive association between exposure to phthalate, or phthalate metabolites, and the risk of endometriosis (Kim et al., 2010; Scsukova et al., 2016). Since the 2000s, studies have been published on the concentration of various phthalate metabolites in human plasma (Cobellis et al., 2003; Reddy et al., 2006), and on urinary concentrations of phthalate monoester metabolites (Barr et al., 2003; Kim et al., 2015). However, there are still some limits to our knowledge of the pathophysiology of phthalates in endometriosis. This review aims to evaluate the risk of exposure to phthalate esters in the development of endometriosis, based on current data.

\section{CELL CULTURE DATA}

By in vitro treatment of endometrial cells with phthalate esters, it can be shown that phthalates are a possible risk factor for endometriosis. A cell viability assay was performed on both DEHPtreated Ishikawa cells and endometrial stromal cells (ESCs), and the study showed increased viability and resistance to a toxic level of hydrogen peroxide. This supports the pathological theory that DEHP exposure to endometrial cells can increase their viability, thus promoting endometriosis outside the uterus, under stress of the immune cell response (Kim et al., 2010).

In another cell culture study, Wang et al. (2010) proved that DEHP and mono-2-ethylhexyl phthalate (MEHP) impaired the secretion of prostaglandin (PG) from ESCs and oxytocin from ovarian cells. When ESCs were cultured with different levels of DEHP and MEHP, PGF2 $\alpha$ increased proportionally, but PGE2 decreased. Granulosa and luteal cells (from ovarian follicles) were treated with DEHP and MEHP, which was found to increase oxytocin secretion significantly. This study suggested that the levels of prostaglandin and oxytocin, which are estradioldependent hormones, are altered by phthalate esters, and this could affect the estrous cycle. Further studies are needed to elucidate the association between prostaglandins, oxytocin and endometriosis. Also, in this study, DEHP- and MEHP-treated ESCs and ovarian cells did not suffer reduced viability in the MTT assay, which seems to be consistent with the previous study by Kim et al. (2010).

Oxidative stress is known to be associated with endometriosis (Carvalho et al., 2012), and a study has demonstrated that DEHP causes oxidative stress in ESCs, in vitro (Cho et al., 2015). ESCs were stained with the peroxide-sensitive fluorescent probe, DCF-DA (2,7-dichlorofluorescein diacetate) and found significantly increased DCF fluorescence in cells treated with DEHP, which signifies the generation of reactive oxygen species (ROS). Concurrently, real-time PCR was used to show a decrease in antioxidant enzymes, such as superoxide dismutase (SOD), glutathione peroxidase (GPx), heme oxygenase (HO), and catalase (CAT). This study also elucidated the molecular mechanism leading to oxidative stress, by detecting increases in the signaling pathways of mitogen-activated protein kinase (MAPK) and nuclear factor kappa-light-chain-enhancer of activated B cells (NF-kB), following DEHP exposure. Since oxidative stress is associated with 
increased ROS, the disruption of defense mechanisms (antioxidant enzymes), and activated MAPK/NF-kB signaling (Klaunig et al., 2010), this study indicates that DEHP causes oxidative stress in ESCs, which results in endometriosis. In addition, as DEHP treatment also increased mRNA expression of estrogen receptor- $\alpha$ (ER- $\alpha$ ) in ESCs, the study suggested that the change in ER- $\alpha$, triggered by DEHP, could be the initial mediator of MAPK/NF-kb signaling.

Based on the previous model, further studies were conducted using DEHP and various biological indicators that may cause endometriosis. Matrix metalloproteinases (MMPs) are enzymes that regulate the physiological changes of endometrial cycling, by vascular formation and remodeling, and are known to increase their activity in endometriosis (Chen et al., 2013). Several studies have confirmed that the expression of MMP-2 and MMP-9 increases in ectopic and eutopic endometrial tissue of patients with endometriosis (Chung et al., 2002; Collette et al., 2006). Our own in vitro study demonstrated that MMP-2 and MMP-9 activities were significantly increased in DEHP-treated Ishikawa cells and ESCs (Kim et al., 2015). In addition, we found that DEHP exposure increased the phosphorylation of extracellular signal-regulated kinase (Erk) and the expression of $\mathrm{p} 21$-activated kinase 4 (Pak4). Both of these biological indicators are assumed to be related to endometriosis by increasing cell proliferation and resistance to apoptosis (Murk et al., 2008; Kim et al., 2013). Our study also used the invasion assay to show that DEHP increased cellular invasiveness.

Cell culture studies provide a possible pathogenesis, that phthalate causes endometriosis by altering various biological markers and increasing cell viability to penetrate the pelvic cavity.

\section{ANIMAL DATA}

Animal models have been used to provide a basis for how phthalates might disrupt the female reproductive system. Exposure to DEHP has been shown to change the level of serum estradiol, to increase ovarian and uterine weights, and to alter the number of ovarian follicles (Tomonari et al., 2006; Hannon et al., 2014). Such disruption of the endocrine mechanism can induce various female reproductive disorders, such as anovulation, polycystic ovaries, infertility and abortion (Davis et al., 1994; Lovekamp-Swan \& Davis, 2003; Schmidt et al., 2012). It is only recently that studies of endometriosis have been conducted in animals.

To our knowledge, our study published in 2015 is the first animal study to reveal the relationship between phthalates and endometriosis (Kim et al., 2015). Ovariectomized mice were implanted with fragments of human endometrial tissue, into the end of the caudal superficial epigastric vein (located on the abdominal wall). After two weeks of treatment with DEHP or vehicle alone, the resultant ectopic, peritoneal endometriotic lesions were of significantly higher volume in DEHPtreated mice compared to the control group. Also, in endometrial tissue of DEHP-treated mice, biological markers increased indicating cellular proliferation and invasiveness, such as MMP-2, 9 and Pak4, were increased. This study provided clear evidence that DEHP causes endometriosis.

In another mouse study, using uterine tissue sections, DEHP was found to alter uterine functions (Richardson et al., 2018). In this study, mice were exposed to different concentrations of DEHP for 30 days. DEHP caused the dilation of endometrial blood vessels and increased the proliferation of ESCs, at almost all dosages $(200 \mu \mathrm{g} / \mathrm{kg} /$ day $-200 \mathrm{mg} / \mathrm{kg} /$ day $)$. At the highest dosage $(200 \mathrm{mg} / \mathrm{kg} /$ day) of DEHP, the number of uterine glands increased. However, a low dosage (200 $\mu \mathrm{g} / \mathrm{kg} / \mathrm{day}$ ) reduced the proliferation of ESCs. This result suggested that DEHP, at various exposure levels, can adversely affect uterine and endometrial cell function.

A recent study has been conducted on chronic, low-dose DEHP exposure in mice (Kim et al., 
2019). Mice were given diluted doses of DEHP (133 or 1,330 $\mu \mathrm{g} / \mathrm{L}$ ) in drinking water, for a period of 10 weeks. Later, their uteri were dissected, and ESCs were found to have significantly increased in both DEHP-administered groups. This study also showed that localization of steroid hormone receptors in endometrial tissue was changed by DEHP.

The previous two studies are not directly related to DEHP and endometriosis, but have proved that DEHP causes a change in the endometrial environment. Considering that endometriosis is caused by altered ectopic endometrial cells, these animal studies may provide a basis for the notion that DEHP can cause endometriosis.

\section{HUMAN DATA}

Many studies have been published on the concentrations of various phthalate metabolites in human plasma and urine (Table 1). In 2003, a study was published of the association between plasma concentrations of phthalate esters and the occurrence of endometriosis in women (Cobellis et al., 2003). Plasma concentrations of DEHP were significantly higher in patients with endometriosis than in controls, and $92.6 \%$ of patients showed detectable DEHP and MEHP levels in peritoneal fluid, obtained during surgery. Since then, some studies have measured DEHP in blood samples from patients with endometriosis. Reddy et al. (2006) discovered an increase in the level of various phthalate esters, including DEHP, in infertile women with endometriosis. In addition, Kim et al. (2011) found that plasma concentrations of MEHP, the main hydrolysis metabolite of DEHP, were significantly high in patients with advanced-stage endometriosis. In this prospective case-control study, all 266 patients have undergone gynecological benign surgery, of which 97 patients have been diagnosed with endometriosis stage 3 or stage 4, and 197 patients have been assigned to control group without endometriosis. Patients with minimal or mild endometriosis and adenomyosis were excluded. In this study, MEHP and DEHP levels were significantly high in

Table 1. Characteristics of the studies of human data

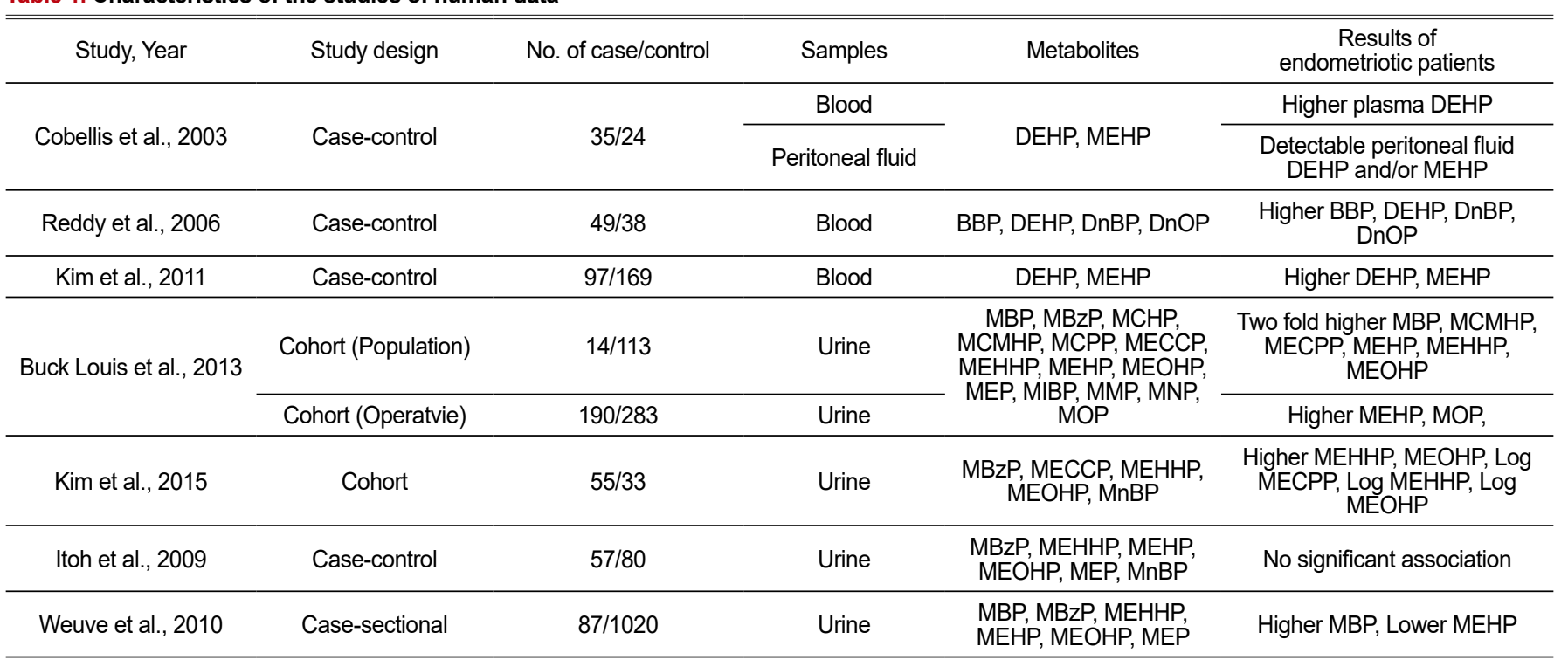

DEHP, di-2-ethylhexyl phthalate; BBP, butyl benzyl phthalate; DnBP, di-n-butyl phthalate; DnOP, di-n-octyl phthalate; MBP, mono-n-butyl phthalate; MBzP, monobenzyl phthalate; MCHP, monocyclohexyl phthalate; MCMHP, mono-2-carboxymethyl hexyl phthalate; MCPP, mono-3-carboxypropyl phthalate; MECCP, mono-2-ethyl-5-carboxyphentyl phthalate; MEHHP, mono-2-ethyl-5-hydroxyhexyl phthalate; MEHP, mono-2-ethylhexyl phthalate; MEOHP, mono-2-ethyl-5-oxohexyl phthalate; MEP, monoethyl phthalate; MIBP, mono-2-isobutyl phthalate; MMP, monomethyl phthalate; MnBP, mono-n-butylphthalate; MNP, monoisonoyl phthalate; MOP, monooctyl phthalate. 
the endometriosis group, but there was no difference between stage 3 and stage 4 of endometriosis. Also, in a control group, there was no difference in MEHP or DEHP between subgroups.

However, these studies on the use of blood samples have limitations, caused by the potential for laboratory contamination with phthalates while using medical devices such as intravenous medical tubing and blood storage bags. Phthalate diesters are rapidly hydrolyzed to monoesters and then excreted in urine, via various oxidative metabolisms. Studies have shown that oxidized metabolites of DEHP, such as mono-(2-ethyl-5-hydroxyhexyl) phthalate (MEHHP) and mono-(2-ethyl5-oxohexyl) phthalate (MEOHP), are detected in urine at higher concentrations than MEHP (Barr et al., 2003; Kato et al., 2004). Based on several studies, the Centers for Disease Control and Prevention $(\mathrm{CDC})$ introduced urinary phthalate metabolites as a sensitive biomarker for exposure to phthalate diester (CDC, 2009).

A large, multicenter cohort study was conducted to reveal the relationship between the phthalate metabolites in urine and endometriosis. This study found a twofold or higher urine concentration for six phthalate metabolites, including MEHP, MEHHP, and MEOHP, in the population cohort ( $\mathrm{n}=131)$, where endometriosis was diagnosed with magnetic resonance imaging (MRI). Two phthalates, monooctyl phthalate (MOP) and MEHP, were found to be increased in the operative cohort ( $\mathrm{n}=495)$ when undergoing surgery (Buck Louis et al., 2013). In our recent prospective cohort study we included 55 patients who underwent surgery and were diagnosed with advanced stage endometriosis, and we also found that MEHHP and MEOHP were associated with endometriosis (Kim et al., 2015). However, some studies have shown an inverse association between some urinary metabolites and endometriosis (Itoh et al., 2009; Weuve et al., 2010). Cai et al. (2019) published a meta-analysis of the association between five phthalate metabolites and endometriosis, which reviewed eight studies and concluded that MEHHP was associated with endometriosis, especially in Asia, whereas four other metabolites, including MEOHP and MEHP, were not (Cai et al., 2019). However, this meta-analysis had some limitations, as it included studies analyzing different types of phthalate metabolites, some of which had inadequate control selections. More research on urine samples should be done, to prove the relationship between phthalate and endometriosis, and to find sensitive biomarkers.

\section{CONCLUSION}

In this review, the possible association between phthalate esters and endometriosis has been discussed, using evidence from studies carried out in cell culture, in animal models and in humans. Based on a number of studies, a potential mechanism of action for phthalate esters on cells can be seen as involving oxidative stress, inflammatory enzymes, and hormone receptors in cell membranes (Fig. 1). It is also assumed that these interrelated signals increase the viability, resistance and proliferation of cells, causing endometriosis, but there are still many unknown elements.

People are usually exposed to phthalates through digestion, but this can also occur through skin and inhalation. Ingested phthalates are rapidly broken down to their monoester forms, and excreted quickly in urine. DEHP has a half-life of 12 hours, and is released from the body within 24 hours. However, metabolites that enter the bloodstream circulate in the body and accumulate in some fats, or are secreted in breast milk (ATSDR, 2002). They are detected not only in blood or urine but also in amniotic fluid and in the placenta that directly can affect the fetus (Swan, 2008). Therefore, the risk of exposure to phthalate in fetal life might be more important than exposure in adult life. Further studies are necessary to identify long-term effects of phthalate based on the fetal exposure data as well as those from adolescents and adults. 


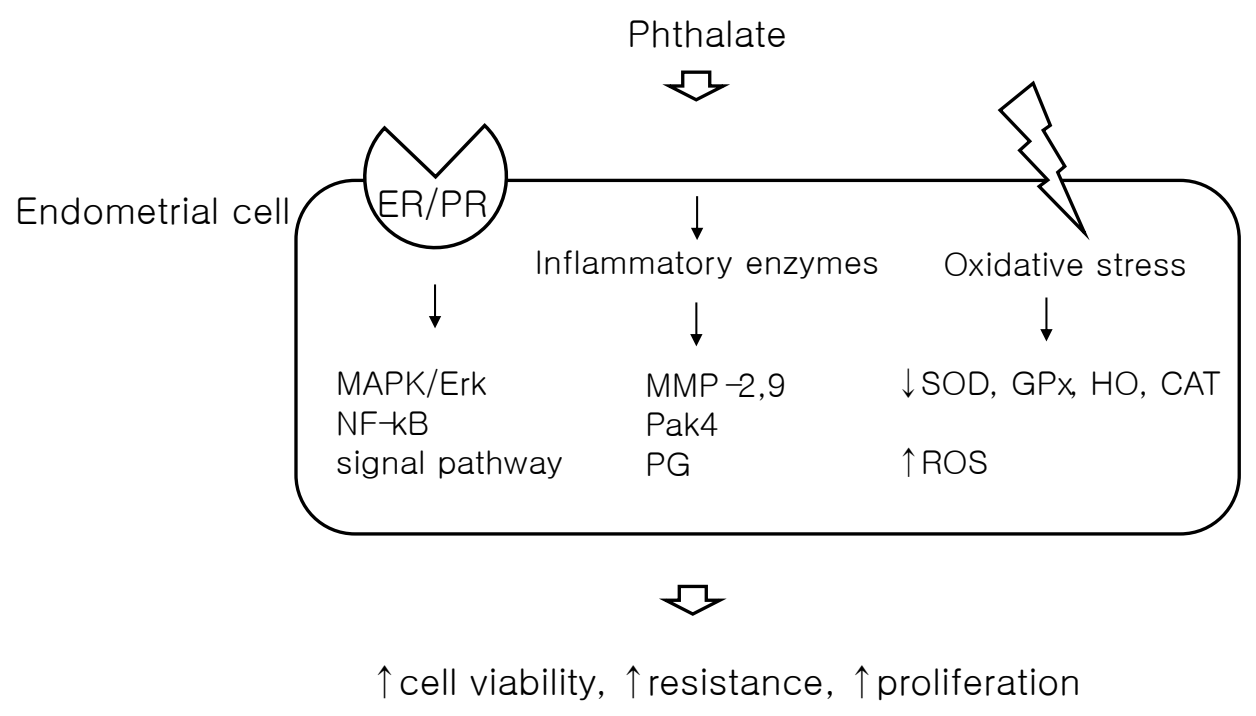

Fig. 1. Potential mechanisms of phthalate esters on endometrial cells. DEHP acts on hormone receptors, such as ER and PR, and activates several inflammatory enzymes. These change the signaling pathways including MAPK/Erk and NF-kB, and release enzymes such as MMP-2/9, Pak4 and PG. Also, DEHP causes oxidative stress, reduces antioxidant enzymes such as SOD, GPx, HO, and CAT, and increases ROS. The interaction of all these changes increases the viability, resistance and proliferation of cells outside the uterus causing endometriosis. DEHP, di-2-ethylhexyl phthalate; ER, estrogen receptor; PR, progesterone receptor; MAPK, mitogen-activated protein kinase; Erk, extracellular signalregulated kinase; NF-kB, nuclear factor kappa-light-chain-enhancer of activated B cells; MMP, matrix metalloproteinase; Pak4, p21-activated kinase 4; PG, prostaglandin; SOD, superoxide dismutase; GPx, glutathione peroxidase; HO, heme oxygenase; CAT, catalase; ROS, reactive oxygen species.

\section{REFERENCES}

Agency for Toxic Substances and Disease Registry [ATSDR] (2002) Toxicological Profile for Di(2-ethylhexyl)phthalate. ATSDR, Atlanta, GA, USA.

Barr DB, Silva MJ, Kato K, Reidy JA, Malek NA, Hurtz D, Sadowski M, Needham LL, Calafat AM (2003) Assessing human exposure to phthalates using monoesters and their oxidized metabolites as biomarkers. Environ Health Perspect 111:1148-1151.

Buck Louis GM, Peterson CM, Chen Z, Croughan M, Sundaram R, Stanford J, Varner MW, Kennedy A, Giudice L, Fujimoto VY, Liping Sun MS, Wang L, Guo Y, Kannan K (2013) Bisphenol A and phthalates and endometriosis: The endometriosis: Natural history, diagnosis and outcomes study. Fertil Steril 100:162-169.

Cai W, Yang J, Liu Y, Bi Y, Wang H (2019) Association between phthalate metabolites and risk of endometriosis: A meta-analysis. Int J Environ Res Public Health 163678.

Carvalho LFP, Samadder AN, Agarwal A, Fernandes LFC, Abrão MS (2012) Oxidative stress biomarkers in patients with endometriosis: Systematic review. Arch Gynecol Obstet 286:10331040.

Centers for Disease Control and Prevention [CDC] (2009) Fourth national report on human exposure to environmental chemicals. U.S. Department for Health and Human Services, Atlanta, GA, USA. Available from https://www.cdc.gov/exposurereport. Access at Jun 30, 2020.

Chen Q, Jin M, Yang F, Zhu J, Xiao Q, Zhang L (2013) Matrix metalloproteinases: Inflammatory regulators of cell behaviors in vascular formation and remodeling. Mediators Inflamm 
2013:928315.

Cho YJ, Park SB, Han M (2015) Di-(2-ethylhexyl)-phthalate induces oxidative stress in human endometrial stromal cells in vitro. Mol Cell Endocrinol 407:9-17.

Chung HW, Lee JY, Moon HS, Hur SE, Park MH, Wen Y, Polan ML, (2002) Matrix metalloproteinase-2, membranous type 1 matrix metalloproteinase, and tissue inhibitor of metalloproteinase-2 expression in ectopic and eutopic endometrium. Fertil Steril 78:787-795.

Cobellis L, Latini G, De Felice C, Razzi S, Paris I, Ruggieri F, Mattzeo P, Petraglia F (2003) High plasma concentrations of di-(2-ethylhexyl)-phthalate in women with endometriosis. Hum Reprod 18:1512-1515.

Collette T, Maheux R, Mailloux J, Akoum A (2006) Increased expression of matrix metalloproteinase-9 in the eutopic endometrial tissue of women with endometriosis. Hum Reprod 21:3059-3067.

Davis BJ, Maronpot RR, Heindel JJ, (1994) Di-(2-ethylhexyl) phthalate suppresses estradiol and ovulation in cycling rats. Toxicol Appl Pharmacol 128:216-223.

Frederiksen H, Skakkebaek NE, Andersson AM (2007) Metabolism of phthalates in humans. Mol Nutr Food Res 51:899-911.

Hannon PR, Peretz J, Flaws JA (2014) Daily exposure to di(2-ethylhexyl) phthalate alters estrous cyclicity and accelerates primordial follicle recruitment potentially via dysregulation of the phosphatidylinositol 3-kinase signaling pathway in adult mice. Biol Reprod 90:136.

Herreros-Villanueva M, Chen CC, Tsai EM, Er TK (2019) Endometriosis-associated ovarian cancer: What have we learned so far? Clin Chim Acta 493:63-72.

Itoh H, Iwasaki M, Hanaoka T, Sasaki H, Tanaka T, Tsugane S (2009) Urinary phthalate monoesters and endometriosis in infertile Japanese women. Sci Total Environ 408:37-42.

Jarfelt K, Dalgaard M, Hass U, Borch J, Jacobsen H, Ladefoged O (2005) Antiandrogenic effects in male rats perinatally exposed to a mixture of di(2-ethylhexyl) phthalate and di(2-ethylhexyl) adipate. Reprod Toxicol 19:505-515.

Kato K, Silva MJ, Reidy JA, Hurtz D $3^{\text {rd }}$, Malek NA, Needham LL, Nakazawa H, Barr DB, Calafat AM (2004) Mono(2-ethyl-5-hydroxyhexyl) phthalate and mono-(2-ethyl-5-oxohexyl) phthalate as biomarkers for human exposure assessment to di-(2-ethylhexyl) phthalate. Environ Health Perspect 112:327-330.

Kim J, Cha S, Lee MY, Hwang YJ, Yang E, Choi D, Lee SH, Cheon YP (2019) Chronic and low dose exposure to nonlyphenol or di(2-ethylhexyl) phthalate alters cell proliferation and the localization of steroid hormone receptors in uterine endometria in mice. Dev Reprod 23:263275.

Kim SH, Cho SH, Ihm HJ, Oh YS, Heo SH, Chun S, Im H, Chae HD, Kim CH, Kang BM (2015) Possible role of phthalate in the pathogenesis of endometriosis: In vitro, animal, and human data.J Clin Endocrinol Metab 100:E1502-E1511.

Kim SH, Chun S, Jang JY, Chae HD, Kim CH, Kang BM (2011) Increased plasma levels of phthalate esters in women with advanced-stage endometriosis: A prospective case-control study. Fertil Steril 95:357-359.

Kim SH, Kim SR, Ihm HJ, Oh YS, Chae HD, Kim CH, Kang BM (2013) Regulation of P21activated kinase- 4 by progesterone and tumor necrosis factor-alpha in human endometrium and its increased eduxpression in advanced-stage endometriosis. J Clin Endocrinol Metab 98:E238-E248.

Kim YH, Kim SH, Lee HW, Chae HD, Kim CH, Kang BM (2010) Increased viability of endometrial cells by in vitro treatment with di-(2-ethylhexyl) phthalate. Fertil Steril 94:24132416. 
Kitawaki J, Kado N, Ishihara H, Koshiba H, Kitaoka Y, Honjo H (2002) Endometriosis: The pathophysiology as an estrogen-dependent disease. J Steroid Biochem Mol Biol 83:149-155.

Klaunig JE, Kamendulis LM, Hocevar BA (2010) Oxidative stress and oxidative damage in carcinogenesis. Toxicol Pathol 38:96-109.

Klemmt PAB, StarzinskiPowitz A (2018) Molecular and cellular pathogenesis of endometriosis. Curr Womens Health Rev 14:106-116.

Lovekamp-Swan T, Davis BJ (2003) Mechanisms of phthalate ester toxicity in the female reproductive system. Environ Health Perspect 111:139-145.

Murk W, Atabekoglu CS, Cakmak H, Heper A, Ensari A, Kayisli UA, Arici A (2008) Extracellularly signal-regulated kinase activity in the human endometrium: Possible roles in the pathogenesis of endometriosis.J Clin Endocrinol Metab 93:3532-3540.

Reddy BS, Rozati R, Reddy BV, Raman NV (2006) Association of phthalate esters with endometriosis in Indian women. BJOG 113:515-520.

Richardson KA, Hannon PR, Johnson-Walker YJ, Myint MS, Flaws JA, Nowak RA (2018) Di (2-ethylhexyl) phthalate (DEHP) alters proliferation and uterine gland numbers in the uteri of adult exposed mice. Reprod Toxicol 77:70-79.

Schmidt JS, Schaedlich K, Fiandanese N, Pocar P, Fischer B (2012) Effects of di(2-ethylhexyl) phthalate (DEHP) on female fertility and adipogenesis in $\mathrm{C} 3 \mathrm{H} / \mathrm{N}$ mice. Environ Health Perspect 120:1123-1129.

Scsukova S, Rollerova E, Bujnakova Mlynarcikova A (2016) Impact of endocrine disrupting chemicals on onset and development of female reproductive disorders and hormone-related cancer. Reprod Biol 16:243-254.

Shafrir AL, Farland LV, Shah DK, Harris HR, Kvaskoff M, Zondervan K, Missmer SA (2018) Risk for and consequences of endometriosis: A critical epidemiologic review. Best Pract Res Clin Obstet Gynaecol 51:1-15.

Swan SH (2008) Environmental phthalate exposure in relation to reproductive outcomes and other health endpoints in humans. Environ Res 108:177-184.

Tomonari Y, Kurata Y, David RM, Gans G, Kawasuso T, Katoh M (2006) Effect of di(2ethylhexyl) phthalate (DEHP) on genital organs from juvenile common marmosets: I. Morphological and biochemical investigation in 65-week toxicity study. J Toxicol Environ Health A 69:1651-1672.

Wang X, Shang L, Wang J, Wu N, Wang S (2010) Effect of phthalate esters on the secretion of prostaglandins (F2alpha and E2) and oxytocin in cultured bovine ovarian and endometrial cells. Domest Anim Endocrinol 39:131-136.

Weuve J, Hauser R, Calafat AM, Missmer SA, Wise LA (2010) Association of exposure to phthalates with endometriosis and uterine leiomyomata: Findings from NHANES, 19992004. Environ Health Perspect 118:825-832.

Zondervan KT, Becker CM, Koga K, Missmer SA, Taylor RN, Vigano P (2018) Endometriosis. Nat Rev Dis Primers 4:9.

Zondervan KT, Becker CM, Missmer SA (2020) Endometriosis. N Engl J Med 382:1244-1256. 